\section{La participación social en salud: el desafío de Chile}

\author{
Claudio A. Méndez $y$ \\ Jairo J. Vanegas López ${ }^{2}$
}

Forma de citar: Méndez CA, Vanegas López JJ. La participación social en salud: el desafío de Chile. Rev Panam Salud Publica. 2010;27(2):144-8.

Palabras clave: gestión en salud; participación comunitaria; política de salud; reforma de la atención de salud; Chile; América Latina.

\footnotetext{
1 Instituto de Salud Pública, Facultad de Medicina, Universidad Austral de Chile. La correspondencia debe dirigirse a Claudio A. Méndez, Avenida Senador Acharan Arce s/n, Campus Isla Teja, Universidad Austral de Chile, Valdivia, Chile. Casilla postal: 567. Correo electrónico: claudiomendez@uach.cl

2 Programa de Doctorado en Salud Pública, Facultad de Medicina, Universidad de Chile.
}

En la década de los ochenta, la mayoría de los países de América Latina y el Caribe fueron tanto actores como testigos de un proceso de reformas del sector salud, impulsado principalmente por cambios epidemiológicos, demográficos y sociopolíticos, así como por propuestas de organismos internacionales dirigidas a reducir el gasto en salud (1). En los años noventa, las reformas se orientaron a promover la equidad, la calidad y la eficiencia en la utilización de los recursos, descentralizar los servicios y fortalecer las instancias de participación social en el sector (2). Hasta hoy, sin embargo, las evaluaciones en materia de participación social y su impacto en los sistemas de salud han sido escasas (2).

No fue ajeno a este contexto el sistema de salud de Chile, que en los años ochenta fue protagonista de una de sus reformas más radicales, caracterizada por una fuerte descentralización de la gestión pública que incluyó el establecimiento del Sistema Nacional de Servicios de Salud (SNSS), el traspaso de la atención primaria a los municipios y la creación del Fondo Nacional de Salud (FONASA) y las Instituciones de Salud Previsional (ISAPRE) $(3,4)$. Estos cambios impactaron significativamente en los esquemas de seguro médico y en el acceso a los servicios, además de provocar un severo desfinanciamiento del sector $(5,6)$. Como era de esperar, una vez restablecido el régimen democrático a inicios de los años noventa, los esfuerzos se concentraron particularmente en recuperar la inversión en salud y mejorar la infraestructura y el acceso a los servicios (7).

En 2002, ya en un escenario de mayor inversión, el sistema de salud chileno encaró un nuevo proceso de reforma que incluyó la participación social en salud como uno de sus principios orientadores, aunque en la práctica tal participación no alcanzó a convertirse en un mecanismo real de empoderamiento de la población. Detrás de esta diferencia entre objetivos y resultados existe, antes que nada, un problema de definición.

En efecto, la participación social en salud se define de acuerdo a múltiples dimensiones con diversos actores sociales y contextos sociopolíticos, lo que puede favorecer ambigüedades e incoherencias entre quienes la proponen y quienes la implementan (8). En el caso de Chile, la participación social en salud ha sido definida como un mecanismo de mejoramiento del sector de la salud, convirtiéndola así en una instancia fiscalizadora de la calidad, efectividad y oportunidad de los servicios, así como del uso eficiente de los recursos (9). Esa conceptualización invita a interrogarse acerca de las posibilidades reales de la participación social en salud en Chile tal y como ha sido planteada en el proceso de reforma en curso. 
El presente trabajo, mediante un enfoque de estudio de caso interpretativo, examina la participación social en salud en Chile, comenzando con un análisis del concepto, para luego describir su evolución dentro del marco de la reforma del sector y finalmente plantear algunos de los desafíos que entraña su implementación (10).

\section{¿Qué significa la participación social?}

La declaración de Alma Ata definió a la participación social como una estrategia para garantizar que los servicios de atención primaria brinden soluciones a los problemas de salud de la población (11). No obstante esta definición, la participación social en salud ha sido interpretada desde dos perspectivas: una utilitarista, donde los gobiernos y otros sectores de la sociedad se valen de los recursos disponibles en la comunidad para compensar costos en la provisión de servicios de salud, y otra que la ve como una herramienta de empoderamiento que da a la comunidad un papel más activo y de mayor responsabilidad en lo referido a la salud de la población (12).

Bajo esta última premisa, la participación social se entiende como un proceso en que los miembros de la comunidad, individual o colectivamente, asumen diferentes niveles de compromisos y responsabilidades. La población identifica sus problemas, formula y ofrece soluciones, crea organizaciones para dar continuidad a los programas y en general contribuye a satisfacer las necesidades de salud de una manera deliberada y democrática $(13,14)$.

El concepto de participación social ha mutado hacia una suerte de juego entre consumidores y proveedores, donde los primeros tienen demandas y necesidades que los proveedores de servicios de salud tratan de satisfacer (15). Esta perspectiva discrepa con la participación social vista como la oportunidad de estimular la autonomía local y la participación activa en la producción de salud (16). De allí que el concepto de participación social conlleve la complejidad de convocar distintas visiones para su inclusión en las políticas de salud. Dentro de las posiciones favorables a la inclusión de la participación social en salud, destacan i) la que en su argumento privilegia los aspectos políticos y sociales, cuyo efecto sea democratizador sobre la sociedad; ii) los que señalan los efectos positivos de la participación en la salud de la población, convirtiéndola en necesaria para el éxito de los programas sanitarios, y iii) la que considera a la participación en salud como una vía de manipulación social y política (17).

A principios de la presente década, la participación social en salud era considerada una "función esencial de la salud pública", en el sentido de facilitar el coprotagonismo de la comunidad organizada en los diferentes programas sanitarios y fortalecer la construcción de alianzas intersectoriales con la sociedad civil (18). Sin embargo, en la actualidad es vista desde la perspectiva de la democratización de los servicios de salud, entendida la democratización como un proceso histórico en donde las relaciones entre el estado y la sociedad se vuelven más cercanas y responsables (19).

\section{La participación social en los años ochenta y noventa}

Los procesos de reforma de los últimos 20 años se dieron a lo largo de toda América Latina y el Caribe. En este contexto se iniciaron formulaciones de políticas públicas dirigidas a mejorar los sistemas de salud y sus prestaciones a los diferentes sectores de sus poblaciones. En opinión de algunos autores, la formulación de estos procesos se dio de forma centralizada y al margen parcial o total de mecanismos de participación social (20).

A finales de los años setenta entró en escena la atención primaria de salud con el lema "Salud para Todos en el Año 2000", promulgado como propósito principal en la reunión de Alma-Ata, ${ }^{3}$ donde se asignó una gran relevancia a la participación comunitaria y a la descentralización de los sistemas de salud (21).

Sin embargo, entre la década de los ochenta y principios de los noventa el panorama regional experimentó una transformación con consecuencias sociales importantes. La crisis económica y la insostenible deuda externa obligaron a los países latinoamericanos a adoptar medidas fiscales austeras que se tradujeron en una reducción del gasto público y del papel del Estado en la provisión de servicios sociales $(22,23)$. Paralelamente se dieron transformaciones demográficas y epidemiológicas que cambiaron los perfiles de salud-enfermedad de las poblaciones. Entre los aspectos relevantes de la época sobresale la entrada en el escenario mundial del virus de la inmunodeficiencia humana y el sida (VIH/Sida) (21).

Algunos autores sostienen que pese a estas dificultades, los procesos de reforma sanitaria implementados en esos años han mostrado avances en temas como financiamiento, equidad, eficiencia y desarrollo de esquemas que permiten a las poblaciones participar en el costeo y aseguramiento de los sistemas (24). Sin embargo, diferentes estudios observan que temas como la descentralización de los servicios, supuestamente dirigidos a facilitar a la participación social (25), no han logrado tal objetivo. Se sugiere que el principal problema ha girado alrededor del modo en que la descentralización fue implantada y su tenue impacto en la participación (25-27). Las reformas de la salud de los años noventa propusieron un papel menos relevante del Estado y una mayor validación del modelo de mercado, en virtud del cual el paciente participa como consumidor y como cliente, al seleccionar y evaluar el servicio por el cual paga $(21,28)$.

En este marco, la participación de la ciudadanía en el sistema de salud se limita a aspectos tales como el establecimiento de prioridades y la asignación y distribución de los recursos (28). Más aún, hasta la fecha la población, que debería ser protagonista y es de hecho blanco de las políticas públicas, se encuentra totalmente al margen de la toma de decisiones, frente a lo

\footnotetext{
3 Conferencia Internacional sobre Atención Primaria de Salud de Alma-Ata, realizada en Kazajistán en 1978, organizada por la OMS/OPS y el UNICEF, y patrocinada por la entonces Unión de Repúblicas Socialistas Soviéticas (URSS).
} 
que algunos autores han descrito como un monologo entre quienes formulan las políticas $(21,29)$. Al respecto, evaluaciones de la participación social en Brasil y Colombia identificaron como debilidades el desconocimiento de los mecanismos de participación ciudadana y asimetrías de poder entre el Estado y las organizaciones de la sociedad civil como las principales barreras para garantizar la participación social (30-33).

\section{La reforma y la participación social en Chile}

A inicios de los años setenta, el gobierno de la Unidad Popular enfatizó la transición al socialismo como la vía para impulsar la participación comunitaria $(34,35)$. No obstante, para algunos autores este enfoque estuvo más cercano a la institucionalidad estatal que a la participación (36). Posteriormente, la dictadura militar constituyó un período que privilegió la toma de decisiones a nivel central (34). En los años noventa, con gobiernos democráticos en el poder, la participación social fue parte de los cambios al sistema principalmente a través de la creación del Plan Nacional de Promoción de la Salud $(4,37)$. Sin embargo, algunas de las evaluaciones realizadas a dicho plan han señalado que la existencia de distintas concepciones respecto de lo que significa la participación social es uno de los principales obstáculos para su implementación (38). En el caso de la reforma en curso, la participación social en salud se incluyó como uno de los principios orientadores de su diseño. A su vez, los objetivos de la reforma se dirigieron a definir garantías explícitas y exigibles para los ciudadanos, el establecimiento de solidaridad en el financiamiento de la atención de salud y la mejora de los modelos de atención y gestión del sistema (9).

La ejecución de la reforma comenzó con la promulgación en 2003 de la ley 19 888, cuyo título indica claramente su temática: Establece Financiamiento Necesario para Asegurar los Objetivos Sociales Prioritarios del Gobierno (39). Posteriormente se promulgaron la ley 19937 de Autoridad Sanitaria y Gestión (40), la ley 19966 que establece un Régimen de Garantías en
Salud (GES) (41) y la ley 20015 que modifica la ley de ISAPRES (42) (cuadro 1). Actualmente, la única ley de la reforma que no ha sido promulgada es la concerniente a los Deberes y Derechos de las Personas.

Si bien como principio orientador la participación social en salud atraviesa todos los cuerpos legales de la reforma, es en la ley 19937 de Autoridad Sanitaria y Gestión y el proyecto de Ley sobre Derechos y Deberes de las Personas en donde se explicitan los principales cambios. Mediante la Ley de Autoridad Sanitaria y Gestión se establece la participación de representantes de la comunidad en la integración del Consejo Consultivo que deberá adoptar el director de los Establecimientos de Autogestión en Red. La función de este consejo será establecer un nexo entre la gestión de los establecimientos y las necesidades que la comunidad identifica en el ámbito de la salud. No obstante, no tendrá poder de decisión.

Si bien es por medio de la Ley de Autoridad Sanitaria que se da paso a la participación de la ciudadanía a nivel de la gestión de los establecimientos sanitarios, es en el Proyecto de Derechos y Deberes de las Personas donde se establecen aspectos fundamentales respecto de la relación de los ciudadanos con el sistema de salud. Dentro de los derechos que establece destacan el trato digno, el acceso a la información, la autonomía del usuario (consentimiento informado), regulación a la investigación clínica, consultas y reclamos y participación de los usuarios. Recíprocamente, el proyecto incluye deberes relacionados al respeto por parte de los usuarios hacia el equipo de salud y la responsabilidad que los ciudadanos asumen al momento de solicitar y recibir atención de salud por parte de una institución sanitaria.

\section{Los desafíos de instrumentar la participación social}

Algunos autores han destacado la ausencia de la participación de la ciudadanía en el diseño y el consenso de la reforma en Chile (43). Al mismo tiempo, si bien en la actualidad se han abierto espacios orienta-

CUADRO 1. Contenidos básicos de los cuerpos legales que integran la reforma de salud iniciada en Chile en 2003

\begin{tabular}{|c|c|}
\hline Cuerpo legal & Contenido \\
\hline Ley 19937 (autoridad sanitaria y gestión) & $\begin{array}{l}\text { - Define la nueva estructura del Ministerio de Salud de Chile. } \\
\text { - Crea la Subsecretaría de Redes Asistenciales, la cual coordina toda la red asistencial del país, } \\
\text { y la Subsecretaría de Salud Pública, encargada de las normas sanitarias y la vigilancia } \\
\text { epidemiológica. } \\
\text { - Creación de los Establecimientos de Autogestión en Red, los cuales serán centros de salud } \\
\text { con sistemas de medición de costos, calidad de prestaciones brindadas y satisfacción de los } \\
\text { usuarios. } \\
\text { - Crea la Superintendencia de Salud, que tiene a su cargo la supervigilancia de FONASA y de } \\
\text { cada ISAPRE. }\end{array}$ \\
\hline Ley 19966 (garantías en salud) & $\begin{array}{l}\text { - Establece garantías explícitas en materia de salud, incluidos el acceso, la calidad, la protec- } \\
\text { ción financiera y la oportunidad con que deben ser otorgadas las prestaciones de programas, } \\
\text { enfermedades y condiciones de salud considerados prioritarios. } \\
\text { - Actualmente el número de patologías o intervenciones priorizadas asciende a } 56 \text {, pero se } \\
\text { espera aumentarlo a } 80 \text { como meta para } 2010 \text {. }\end{array}$ \\
\hline
\end{tabular}

Fuente: basado en Méndez CA. Los recursos humanos de salud en Chile: el desafío pendiente de la reforma. Rev Panam Salud Publica. 2009;26(3):276-80. a FONASA: Fondo Nacional de Salud.

bISAPRE: Institución de Salud Previsional. 
dos a la participación social en el sistema sanitario, todavía no se ha logrado la intensidad necesaria, la autonomía de los actores ni la participación suficiente de la comunidad (44). De esta forma, los desafíos participativos consisten en crear espacios para la toma de decisiones dentro de un proceso que conlleva la adopción de nuevos modelos de gestión e intervenciones de salud priorizadas cuya ejecución sea garantizada explícitamente.

Por tal motivo, aun cuando la participación social atraviesa estructuralmente a la reforma de la salud, el lograr posicionarla frente a la prioridad de implementar los cambios organizacionales por parte de los equipos de salud implica definir a la participación más allá de lo conceptual y lo legal. Este sería el modo de lograr que la ciudadanía perciba la influencia que puede llegar a ejercer en la calidad de los servicios que recibe (30), además de persuadir a los equipos de salud respecto de la importancia de la participación cuando se pongan en práctica los cambios organizacionales de la reforma.

Adicionalmente, se hace necesario definir la participación respecto de su fin democratizador dentro del sector, la inclusión y la autonomía de todos los actores que desempeñan un papel y hacen posible un debate en función de la toma de decisiones. De lo contrario, la política de participación social en Chile seguirá siendo una estrategia de descentralización que mantiene las asimetrías de poder observadas en otros países de la Región (31).

Otro reto sustantivo es mejorar la coordinación entre las diferentes instancias comprometidas en la participación, creando espacios, mecanismos y formas de financiamiento que permitan desarrollar y promover una verdadera participación social. La ausencia de dicha coordinación ya ha sido destacada como un obstáculo para la participación en otras experiencias (45, 46). Además, es preciso fortalecer la coordinación relacionada con la generación de evidencias que permitan demostrar el impacto de las distintas estrategias de participación social. Se ha señalado que hay escasos datos probatorios sobre resultados de los diversos enfoques que promueven la participación social y, en particular, sobre el grado en que las poblaciones más vulnerables logran ser escuchadas o representadas en las jerarquías de poder (46).

Es preciso que el sistema de salud chileno entregue a la ciudadanía información idónea que indique la manera de participar tanto individual como colectivamente en el proceso de formulación y ejecución de las políticas públicas, en particular porque la ausencia de tal información ha sido reconocida como una barrera a la participación social en la salud $(32,33)$. Finalmente, y puesto que el presente artículo representa una primera aproximación a la participación social en la re- forma, será relevante profundizar este tema a través de estudios cuantitativos y cualitativos que permitan identificar los factores que influyen en el nivel de la participación y el grado de impacto que esta tiene en el sector de la salud, comparando luego esta experiencia con otras del continente.

\section{Comentarios finales}

La participación social en Chile debe pasar de ser un mecanismo de control social a ser una instancia que permita la inclusión, la autonomía y el debate, de forma tal que las ideas y propuestas de la ciudadanía puedan trascender en mejores políticas públicas de salud. Así, la ventaja de posicionar a la participación social como un proceso de democratización más amplio radicará en contar con un sistema de salud basado en políticas cuya génesis sea establecida entre la ciudadanía y sus equipos locales de salud.

La reforma que tiene lugar hoy en Chile presenta un camino difícil para el futuro de la participación en el sistema de salud, principalmente en razón de la disonancia que existe entre el discurso de las fuentes oficiales y el pragmatismo con que los equipos de salud perciben la inclusión de la sociedad en las decisiones. En este sentido, la democratización que se propone podría consolidar la reforma de la salud manteniendo a la ciudadanía como eje central del cambio.

\section{SYNOPSIS}

\section{Community participation in health: the challenge in Chile}

Health care reforms implemented in Latin America and the Caribbean over the last 20 years have viewed community participation as a system-wide component. Nonetheless, these reform efforts have yet to break through the conceptual and operational barriers holding back the development and expansion of community participation.

In Chile, changes introduced to the health care system are far from achieving any real participation from the community. Therefore, the consumer's role needs to be redefined from merely controlling the parts, to reaching across the whole system in a way that consumer input might identify and quickly correct any posible shortcomings in the health system's design, as well as its operations.

With this in mind, the main challenges are to strengthen coordination among the various promotion and participation commitments, as well as community control, and to generate data and other evidence to assess the impact of community participation in health strategies.

Key words: health management; community participation; health policy; health care reform; Chile; Latin America.

\section{REFERENCIAS}

1. Homedes N, Ugalde A. Why neoliberal health reform have failed in Latin America. Health Policy. 2005;71:83-96.
2. Infante A, de la Mata I, López-Acuña D. Reforma de los sistemas de salud en América Latina y el Caribe: situación y tendencias. Rev Panam Salud Publica. 2000;8(1/2):13-20. 
3. Annick M. The Chilean health system: 20 years of reforms. Salud Publica Me. 2002;44(1):60-8.

4. Azevedo AC. La provisión de servicios de salud en Chile: Aspectos históricos, dilemas y perspectivas. Rev Saude Publica. 1998;32(2):192-9.

5. Hómedes N, Ugalde A. Privatización de los servicios de salud: las experiencias de Chile y Costa Rica. Gac Sanit. 2002;16(1):54-62.

6. Jimenez J, Bossert T. Chile's health sector reform: lessons from four reform periods. Health Policy. 1995;32:155-66.

7. Artaza O, Montt J, Vásquez C. Estructura y recursos humanos en un hospital público chileno: una experiencia de cambio. Rev Panam Salud Publica. 1997;2(5):342-51.

8. Delgado Gallego ME, Vázquez Navarrete ML, Zapata Bermúdez, Hernán García M. Participación social en salud: conceptos de usuarios, líderes comunitarios, gestores y formuladores de políticas en Colombia. Una mirada cualitativa. Rev Esp Salud Publica. 2005;79:697-707.

9. Sandoval H. Mejor salud para los chilenos: fundamentos sanitarios, políticos y financieros de la necesidad y oportunidad de hacer una reforma al sistema de salud chileno. Cuad Med Soc. 2004;43(1):5-20.

10. Lijphart A. Política comparada y método comparado. Revista Latinoamericana de Política Comparada. 2008;(1): 213-42.

11. Gillam S. Is the declaration of Alma Ata still relevant to primary health care? BMJ. 2008;336:536-8.

12. Morgan LM. Community participation in health: perpetual allure, persistent challenge. Health Policy Plan. 2001;16 (3):221-30.

13. Zakus J, Lysack C. Revisiting community participation. Health Policy Plan. 1998;13(1):1-12.

14. OPS. Evaluación para el fortalecimiento de procesos de participación social en la promoción y el desarrollo de la salud en los sistemas locales de salud. Washington DC: OPS; 1994.

15. Martín-García M, Ponte-Mittelbrunn C, Sánchez-Bayle M. Participación social y orientación comunitaria en los servicios de salud. Gac Sanit. 2006;20(1 supl): 192-202.

16. Mosquera M, Zapata Y, Lee K, Arango C, Varela A. Strengthening user participation through health sector reform in Colombia: a study of institutional change and social representation. Health Policy Plan. 2001;16(2 supl): 52-60.

17. Bronfman M, Gleizer M. Participación comunitaria: necesidad, excusa o estrategia? $\mathrm{O}$ de qué hablamos cuando hablamos de participación comunitaria. Cad Saude Publica. 1994;10(1): $111-22$.

18. Muñoz F, López-Acuña D, Halverson P, Guerra de Macedo C, Hanna W, Larrieu $\mathrm{M}$, et al. Las funciones esenciales de la salud pública: un tema emergente en las reformas del sector de la salud. Rev Panam Salud Publica. 2000;8(1/2):126-34

19. Moreira MR, Escorel S. Municipal health councils of Brazil: a debate in the democratization of health in the twenty years of the UHS. Cien Saude Colet. 2009;14(3):795-805

20. Pérez J, Bentacourt J, Suárez P. Descentralización y sistemas de salud en América Latina. Revista de Ciencias Sociales. 2006;12(1):36-45.

21. Vásquez ML, Siqueira E, Kruze I, Da Silva A, Leite IC. Los procesos de reforma y la participación social en salud en América Latina. Gac Sanit. 2002;16 (1):30-8.

22. Vergara C. El contexto de la reformas del sector salud. Rev Panam Salud Publica. 2000;8(1/2):7-12.

23. Almeida C. Reforma de sistemas de servicios de salud y equidad en América Latina y el Caribe: algunas lecciones de los años 80 y 90. Cad Saude Publica. 2002;18(4):905-25.

24. Celedón C, Noé M. Reforma del sector salud y participación social. Rev Panam Salud Publica. 2000;8(1/2):99-104.

25. Ugalde A, Homedes N. Descentralización de los sistemas de salud en América Latina. Gac Sanit. 2002;16(1): 18-29.

26. Homedes N, Ugalde A. Las reformas de salud neoliberales en América Latina: una visión crítica a través de dos estudios de caso. Rev Panam Salud Publica. 2005;17(3):210-20.

27. Ugalde A, Homedes N. La descentralización de los servicios de salud: de la teoría a la práctica. Salud Colectiva. 2008;4(1):31-56.

28. Guzmán Urrea MP. Deficiencias en los diagnósticos de las reformas sanitarias de los años noventa en América Latina. Rev Panam Salud Publica. 2009;25(1): 84-92.

29. Arévalo DA. Participación comunitaria y control social en el sistema de salud. Rev salud publica (Bogota). 2004;6(2): 107-39.

30. Delgado-Gallego ME, Vásquez ML. Percepciones de usuarios y líderes comunitarios sobre su capacidad para influenciar en la calidad de los servicios de salud: un estudio de casos de Colombia y Brasil. Cad Saude Pública. 2009;25(1):169-78.

31. Wendhausen ALP. Relaciones de poder y democracia en los consejos de salud en Brasil: estudio de un caso. Rev Esp Salud Publica. 2006;80:697-704.

32. Delgado-Gallego ME, VázquezNavarrete L. Barreras y oportunidades para la participación social en salud en Colombia: percepciones de los actores principales. Rev salud publica (Bogota). 2006;8(2):128-40.

33. Delgado-Gallego ME, VázquezNavarrete ML. Conocimientos, opiniones y experiencias con la aplicación de las políticas de participación en salud en Colombia. Rev salud publica (Bogota). 2006;8(3):150-67.

34. Labra ME. La reinvención neoliberal de la inequidad en Chile. El caso de la salud. Cad Saude Publica. 2002;18(4): 1041-52.

35. Menanteau-Horta D. El rol del Estado en el desarrollo social y la reforma de la previsión en Chile y EE.UU. Revista Austral de Ciencias Sociales. 2006;10: 05-22.

36. Sepúlveda Álvarez C. Alma Ata-2008, recuerdo y proyección, 30 años después. Cuad Med Soc. 2008;48(4): 251-61.

37. Vío F, Salinas J. Promoción de salud y calidad de vida en Chile: una política con nuevos desafíos. Rev Chil Nutr. 2006;33(1 supl):252-9.

38. Salinas J, Cancino A, Pezoa S, Salamanca F, Soto M. Vida Chile 1998-2006: resultados y desafíos de la política de promoción de la salud en Chile. Rev Panam Salud Publica. 2007;21(2/3): 136-44.

39. Ministerio de Hacienda de Chile. Ley $\mathrm{N}^{\circ}$ 19.888. Establece Financiamiento Necesario para Asegurar los Objetivos Sociales Prioritarios del Gobierno. Santiago: Diario Oficial de la República de Chile; 2003. Hallado en: http://www. bcn.cl/leyes/pdf/actualizado/213493. pdf. Acceso el 10 de octubre de 2009.

40. Ministerio de Salud de Chile. Ley $\mathrm{N}^{\circ}$ 19.937. Autoridad Sanitaria y Gestión. Santiago: Diario Oficial de la República de Chile; 2004. Hallado en: http:// www.bcn.cl/leyes/pdf/actualizado/ 221629. pdf. Acceso el 6 enero de 2009.

41. Ministerio de Salud de Chile. Ley $\mathrm{N}^{\circ}$ 19.966. Régimen General de Garantías en Salud. Santiago: Diario Oficial de la República de Chile; 2004. Hallado en: http://www.bcn.cl/leyes/pdf/ actualizado/229834.pdf. Acceso el 10 de octubre de 2009.

42. Ministerio de Salud de Chile. Ley $\mathrm{N}^{\circ}$ 20.015. Modifica la ley 18.433 de Instituciones de Salud Previsional (ISAPRE). Santiago: Diario Oficial de la República de Chile; 2005. Hallado en: http:/ / www. bcn.cl/leyes/pdf/actualizado/238102. pdf. Acceso el 10 de octubre de 2009.

43. Drago M. La reforma al sistema de salud chileno desde la perspectiva de los derechos humanos. Comisión Económica para América Latina y el Caribe (CEPAL). Serie Políticas Sociales $\mathrm{N}^{\circ}$ 121. Santiago: CEPAL; 2006.

44. Anigstein MS. Participación comunitaria en salud: reflexiones sobre las posibilidades de democratización del sector. Rev Mad. 2008;19:77-88.

45. Carrasco B, Caire V, Sockins E. Evaluation of Senior Citizens' Satisfaction in Primary Health Centres as Assessment of the Academic Model. Education for Health. 2003;16(1):4-13.

46. Murthy RK, Klugman B. Service accountability and community participation in the context of health sector reforms in Asia: implications for sexual and reproductive health services. Health Policy Plan. 2004;19:78-86.

Manuscrito recibido el 16 de abril de 2009. Aceptado para publicación, tras revisión, el 14 de agosto de 2009. 